\title{
Dos primaveras, ¿hacen verano? La institucionalización de la teoría literaria en la Argentina y sus relatos
}

\author{
DIEGO PELLER Universidad de Buenos Aires, Argentina / diegopeller@yahoo.com
}

\section{Resumen}

Este trabajo se propone caracterizar dos momentos clave en el proceso de institucionalización de la teoría literaria como disciplina en la Universidad de Buenos Aires y, debido a su impacto, en las universidades argentinas en un sentido más amplio: la efímera experiencia de 1973-1974 en el marco de las «cátedras nacionales» de la Universidad Nacional y Popular de Buenos Aires, y el seminario «Algunos Problemas de Teoría Literaria» dictado por Josefina Ludmer en 1985 en plena "primavera democrática» y refundación de la carrera de Letras. A partir de una lectura de los trabajos de Leonardo Funes (2009) y Annick Louis (2015) sobre estas dos experiencias emblemáticas, proponemos poner en cuestión las interpretaciones hegemónicas sobre las mismas, así como la posibilidad de fundamentar en narrativas históricas de este tipo un determinado lineamiento con respecto al presente y futuro de la teoría literaria en nuestro país.

Palabras clave: teoría literaria / institucionalización / narrativa histórica / Josefina Ludmer / Universidad de Buenos Aires

\section{The Institutionalization of Literary Theory in Argentina and its Narratives}

Abstract

These paper intends to characterize two key moments in the process of institutionalization of literary theory as a discipline at the Universidad de Buenos Aires and, due to its impact, in the Argentine universities in a broader sense: the ephemeral experience of 1973-1974 within the framework of the «cátedras nacionales» of the Universidad Nacional y Popular de Buenos Aires, and the seminar «Algunos Problemas de Teoría Literaria» dictated by Josefina Ludmer in 1985 in the middle of "primavera democrática» and refounding of the career of Letras. From a reading of the works of Leonardo Funes (2009) and Annick Louis (2015) on these two emblematic experiences, we propose to question the hegemonic interpretations about them, as well as the possibility of grounding in historical narratives of this type a certain guidelines regarding the present and future of literary theory in our country.

Key words: literary theory / institutionalization / historical narrative / Josefina Ludmer / Universidad de Buenos Aires

Recibido: 8/8/2018. Aceptado: 24/9/2018

Para citar este artículo: Peller, Diego (2018). Dos primaveras, ¿hacen verano? La institucionalización de la teoría literaria en la Argentina y sus relatos. El taco en la brea, 8 (junio-noviembre), 138-150. Santa Fe, Argentina: UNL. DOI: 10.14409/tb.vii8.7762 


\section{Introducción. Otoño de la teoría literaria y giro historicista}

En las últimas décadas es posible advertir un giro historicista en el campo de la teoría literaria en la Argentina, como si la disciplina, ante su crisis o su ocaso - dependiendo de las divergentes interpretaciones que se construyen en torno al fenómeno-, se replegara para preguntarse por su propia historia e identidad. Se ha señalado, como un rasgo singular y un tanto enigmático de los estudios literarios en la Argentina — por lo menos desde los años setenta— la paradoja según la cual, por un lado, estos estudios poseen un sesgo marcadamente teórico (se lee y se usa «mucha teoría»), pero, al mismo tiempo, esa pasión por la teoría (Peller 2016) no se habría plasmado en un cuerpo significativo de «obras» teóricas en sentido estricto (no se "produce» una teoría literaria con rasgos propios). A veces este «señalamiento» sufre una inflexión más personalista, y lo que se enfatiza entonces es que los "grandes popes» de la teoría literaria en la Argentina han enseñado, leído y enseñado a leer teoría pero, en tanto sujetos de escritura, han producido fundamentalmente crítica. A lo que me permitiría agregar que, junto con esta producción crítica, no han dejado de proponer diversas interpretaciones históricas sobre los derroteros de la propia disciplina. Se podrían mencionar muchos indicadores de esta torsión autorreflexiva, entre ellos el dossier de la revista El taco en la brea sobre los avatares de la teoría literaria latinoamericana en Argentina, que este trabajo integra, así como el dossier del número 5 (2017) de esta misma publicación, sobre el «Fin y resistencia de la teoría». También la revista Luthor ha dedicado, en los últimos años, un lugar central a la reflexión sobre lo que propuso calificar como el «Eterno ocaso de la teoría» (fue el nombre elegido para el dossier de su número 30, publicado en 2016). Pero el interés de Luthor en el tema supera ampliamente el de ese dossier: la revista, perteneciente al ámbito de la carrera de Letras de la Universidad de Buenos Aires (UBA), desde su inicio se propuso un programa de revisión crítica de la investigación y producción teórica en el marco de la institución. El balance histórico de Luthor tiene entonces como meta instalar en la agenda la necesidad de reformar el actual Plan de Estudios y discutir el lugar que hoy ocupa allí la teoría literaria; no para «eliminarla», pero sí para «repensar» nuevas formas — supuestamente más modernas y productivas- en las que esta podría ofrecerse a los estudiantes, en términos de "metodología», y otras formas de la teoría que, se supone, tendrían una vinculación más evidente con las problemáticas contemporáneas (queer y gender studies, etc.). En este sentido ocupan un lugar clave una serie formada hasta hoy por seis artículos — se trata de un trabajo en progreso- titulados «Aproximaciones a la historia de la teoría literaria en la carrera de Letras de la UBA» (partes I a III por Gustavo Riva y Juan Manuel Lacalle; partes IV y V por Lacalle y Majo Migliore; parte VI por Lacalle y Bogado), ya que en ellos es donde esta agenda se sustenta en términos de una narrativa histórica. No voy a detenerme ahora en estos artículos, lo he hecho en una publicación anterior (2017), pero me parece importante volver a señalar el modo en que se articula en ellos un determinado relato sobre el pasado de la disciplina con una agenda de intervención con relación al futuro de la misma. Lo que importa señalar en este punto, ya que se vincula con el argumento central del presente trabajo, no es qué contenidos específicos asume esa narrativa, ni cuál es esa agenda, sino el presupuesto que subyace a estas intervenciones de Luthor: que es posible y conveniente fundamentar, en la construcción de una narrativa sobre el pasado de la disciplina, una agenda política de intervención y transformación institucional de la misma (que se presenta así como conclusión necesaria de ese balance histórico y no como expresión contingente del deseo de un conjunto de sujetos en el presente). 
Otro fuerte indicador del mencionado giro historicista es el fenómeno editorial de la publicación de clases, en algunos casos de cursos que fueron adquiriendo con los años un estatuto casi mítico, como el seminario de Josefina Ludmer "Algunos Problemas de Teoría Literaria», publicado con el título Clases 1985, treinta años después (Ludmer 2015). Y el primer seminario de Ricardo Piglia dictado en la UBA en 1990, y publicado pocos meses después de las clases de Ludmer (Piglia 2016). Podríamos agregar, aunque es bastante anterior en el tiempo, la publicación de las clases de Aníbal Ford (2005). Fenómeno editorial que ha venido acompañado por un creciente interés por estas clases como objeto de investigación académica y de reflexión crítica. Estoy pensando en los artículos publicados en Luthor ya mencionados (que se detienen especialmente en los programas de los cursos), pero también en los trabajos de Analía Gerbaudo (2011, 2013, 2015, 2016), en el prólogo de Annick Louis (2015) a la edición de las clases de Ludmer, así como en dos textos de tono más testimonial, de Miguel Vitagliano (2011) y Leonardo Funes (2009), sobre los inicios de la teoría literaria como curso curricular en la Facultad de Filosofía y Letras de la UBA.

En el presente trabajo me propongo analizar las operaciones discursivas que construyen, en los textos que integran este corpus, una determinada narrativa histórica sobre los procesos de constitución de la teoría literaria como disciplina. Con este objetivo voy a detenerme especialmente en los textos de Funes y Louis previamente mencionados.

\section{Primera estación: una «primavera interrumpida» en los setenta}

Durante los días 3 y 4 de diciembre de 2009 se celebraron en la Facultad de Filosofía y Letras de la UBA las I Jornadas de Historia de la Crítica en la Argentina. El contexto más inmediato de estas Jornadas era un momento de renovación institucional del Departamento de Letras, cuya marca más notoria era el alejamiento de Jorge Panesi del cargo de Director de dicho Departamento, cargo que pasó a ser ejercido por Américo Cristófalo. No se trataba de cualquier sucesión: Panesi había sido Director del Departamento, con algunas interrupciones, desde la refundación de la carrera tras la última dictadura cívico-militar, de manera que su alejamiento de la dirección marcaba el final de toda una etapa en la historia de la carrera. Por eso resulta particularmente significativo que uno de los primeros emprendimientos del Departamento, bajo la nueva dirección, haya sido proponer un encuentro académico que tuviera como eje la historia de la crítica, como si propusiera ejercitar una suerte de balance histórico colectivo. En idéntica dirección se puede leer un breve volumen publicado también por la Facultad, poco después, en el año 2011, bajo el título Perspectivas actuales de la investigación literaria, en el que cinco profesores, que habían asumido recientemente la dirección de sus respectivas cátedras, en un momento de «recambio generacional en el cuerpo de profesores a cargo de materias de las carrera de grado», reflexionaban sobre «las condiciones, marcos conceptuales, modalidades, intereses y objetivos de la investigación en torno al fenómeno literario que se hace en nuestra Facultad» (Ciordia y otros:5). Pero, nuevamente, este «recambio generacional» asumía características muy singulares: por un lado, podría aducirse, era completamente «normal», respondía a una lógica de funcionamiento de las instituciones según la cual, siguiendo reglas establecidas, en determinado momento los miembros de las nuevas generaciones reemplazan a los de las anteriores en los cargos de dirección. Pero en el caso de la carrera de Letras de la UBA justamente que este recambio «normal» sucediera — y que sucediera más o menos simultáneamente en varias cátedras - era inusual y se constituía en un verdadero acontecimiento, dado que las figuras que eran reemplazadas habían estado a cargo de sus respectivos 
cursos desde la refundación de la «universidad de la democracia» en los tempranos ochenta. Así, Américo Cristófalo, al hacerse cargo de la Cátedra de Literatura del Siglo XIX, ocupaba el lugar de María Teresa Gramuglio, y Miguel Vitagliano, al tomar a su cargo la Cátedra de Teoría Literaria III ocupaba el lugar de Nicolás Rosa, por mencionar dos casos paradigmáticos.

En este contexto, no es extraño que algunas de las intervenciones que se produjeron en el marco de las jornadas y el libro mencionados se hayan detenido, justamente, en los años 1984-1985, el momento de refundación de la carrera. Un momento que se conoce como "primavera democrática» 0 «primavera alfonsinista». Tampoco resulta del todo extraño que el recuerdo de esa primavera haya conducido a la rememoración de otra, la breve "primavera camporista», diez años antes.

Detengámonos en dos de esas intervenciones, una perteneciente al libro y otra a las jornadas, aquellas que se centran en la constitución de la Teoría Literaria como disciplina en la carrera. La primera es la de Miguel Vitagliano y se titula «Variaciones sobre un punto. Notas de trabajo sobre teoría y crítica literaria». Vitagliano comienza su intervención recordando:

Recién a mediados de la década del ochenta, una vez terminada la dictadura, la teoría literaria logró tener nombre de asignatura en las universidades del país. En la Facultad de Filosofía y Letras de la Universidad de Buenos Aires (UBA) fue lo que ocurrió con la cátedra Teoría y Análisis Literario de Enrique Pezzoni y Jorge Panesi, a partir de 1984, y con el seminario "Algunos problemas de Teoría Literaria» de Josefina Ludmer, en 1985, y que desde el año siguiente se convirtió en la materia Teoría Literaria II. (123)

Se trata de un relato fundacional ampliamente difundido. Lo volveremos a encontrar en otros textos. Me interesa detenerme en el matiz temporal que introduce el adverbio «recién». Fue «recién» a mediados de los ochenta cuando la teoría "logra tener nombre de asignatura» en las universidades argentinas. Lo que implica que antes la teoría ya existía (como práctica, como ámbito de discusión, como espacio de trabajo y de saber sobre la literatura); existía pero no tenía nombre, existía sin «todavía» tener nombre, en una suerte de demora que implicaría una ofensa, un daño, una desconsideración a eso que se practicaba y existía como teoría pero que «no había logrado tener nombre de asignatura». Esa situación violenta e injusta «recién» se habría saldado a mediados de los ochenta, una vez terminada la dictadura. Se postula entonces, implícitamente, una suerte de historia paralela, contra-fáctica, en la cual, de no haber sido por la dictadura, la teoría literaria como tal, en tanto tal, hubiera tenido nombre, hubiera logrado tener nombre - y nombre de asignatura - antes, mucho antes, en el momento justo, de manera «natural». Esta interrupción del curso histórico "normal», que habría impedido que la teoría se institucionalizara en su momento justo, y que habría llevado a que «recién» lo hiciera en 1984-1985, sería uno de los factores que explicaría el inusual entusiasmo que la disciplina despertó en el momento de su tardío estallido democrático, pero también explicaría algunas de las falencias, debilidades o «atrasos» que caracterizarían los desarrollos locales de la disciplina. Otro punto que no dejará de volver en las diversas versiones de este momento fundacional.

Pero hay otra primavera, otra fundación mítica de la teoría literaria en la Argentina, anterior en algunos años y que habría quedado sepultada en el olvido para "recién» una vez terminada la dictadura reemerger y finalmente «lograr su nombre», un nombre que había comenzado a articularse diez años atrás, pero que había quedado interrumpido por el invierno abrupto y asesino de la dictadura. 
A ese momento previo se refiere, de manera sumamente sentida, Leonardo Funes en su contribución a las Jornadas de Historia de la Crítica, una contribución que lleva el significativo título «Teoría literaria: una primavera interrumpida en los años setenta» (2009). El trabajo comienza citando el fragmento del artículo de Vitagliano en el que nos hemos detenido previamente. Se refiere a él como «un trabajo reciente, preparado para un libro de próxima aparición» en el que Vitagliano «hace una reseña de la emergencia y consolidación de los estudios de Teoría Literaria en la universidad argentina a partir del regreso de la democracia o, como cada vez más se insiste en denominarlo, con más precisión, en el período de la posdictadura» (79).

Luego de citar a Vitagliano, Funes enuncia el propósito de su propia contribución:

Voy a referirme a un antecedente lejano de la materia [Teoría Literaria] que tuvo lugar en el año 1974. Mediante la Resolución Nº 304 del 15 de marzo de 1974, firmada por el Delegado Interventor Justino M. O’Farrell y por el secretario de Asuntos Académicos Ricardo D. Sidicaro, y por iniciativa del Director del Departamento, el poeta Paco Urondo, se estableció el cambio de nombre de algunas materias. Así, Teoría Literaria I (anual) reemplazaba a Introducción a la Literatura. Se encomendaba al prof. Octavio Prenz, en su carácter de profesor adjunto ordinario del Departamento de Lenguas y Literaturas Modernas, que se hiciera cargo del dictado de Teoría Literaria I. En la misma resolución se nombraba como adjunta de la misma materia a la prof. Hortensia Lemos. (79)

El trabajo de Funes se presenta entonces, desde el vamos, como una continuación del de Vitagliano, pero también como una corrección: no es del todo exacto, acota Funes, que «recién en los ochenta» la Teoría Literaria haya logrado «tener nombre de asignatura»; existió una efímera cátedra de Teoría Literaria I en 1974, aunque es cierto que, desde el punto de vista institucional, se trató de una experiencia violentamente interrumpida y nunca retomada, como si jamás hubiese existido. En este punto, la contribución de Funes se ofrece como un testimonio: su propósito no es someter a ningún tipo de lectura crítica la experiencia interrumpida del dictado de esa cátedra setentista de Teoría Literaria I, sino fundamentalmente testimoniar su acontecer; rescatar una experiencia olvidada enmendando así la omisión de Vitagliano. El punto de vista en el que se sitúa Funes para enunciar no es el del profesor (aunque él sea, en el momento de su intervención, profesor de Literatura Española Medieval, y de hecho sea uno de los colaboradores del volumen colectivo donde se publica el texto de Vitagliano) sino el del alumno entusiasta, que recuerda incluso detalles mínimos, con un grado de precisión que da cuenta de la fuerte carga emotiva que permanece ligada a ese recuerdo, como si lo que Funes está contando en realidad hubiera ocurrido ayer:

Desde ya, soy incapaz de un comentario objetivo o realmente informado de esta experiencia académica. Solo me atrevo a dar testimonio de mi propia percepción y participación como alumno en esa experiencia y su significación en lo que hace al derrotero de mi propia reflexión y posicionamiento ante lo que entendemos como Teoría Literaria. (79)

Funes rememora esa experiencia y se refiere al programa de la materia, luego al contexto histórico: «El contexto histórico no era precisamente la primavera camporista sino más bien el otoño del patriarca, es decir, los últimos meses del General» (80). Destaca a continuación el carácter renovador de la experiencia, tanto en lo que hace a los contenidos que el programa proponía, como 
a la metodología de enseñanza y de evaluación. En cuanto a los contenidos, el programa de la materia, que Funes transcribe en su artículo, muestra claramente la impronta de la Universidad Nacional y Popular (ver Buchbinder:202-207). Es el momento de las llamadas «cátedras nacionales» y en sintonía con ese proyecto, tras dos unidades «introductorias» de carácter más general («I. Concepto de cultura. Definición del objeto literario; II. Consideración de modelos de análisis literario: el formalismo ruso, el estructuralismo, el grupo Tel Quel, la crítica psicoanalítica y la crítica arquetípica, la crítica sociológica, política, histórica»), el curso proponía una orientación muy definida en sus últimos dos puntos: «III. La literatura nacional y popular; IV. Valoración de los modelos de análisis que resulten útiles para el estudio literario en el tercer mundo» (Funes 80). Este programa, sin embargo, no llegaría a cumplirse, como anota Funes:

La historia nos pasó por encima y el curso se interrumpió luego de los exámenes parciales de fin del cuatrimestre, cuando la intervención de Ottalagano, del gobierno de Isabel Perón, cerró la universidad y la Triple A se encargó de enviar al exilio (exterior o interior) al grueso del cuerpo de profesores que había llegado en 1973 para renovar la carrera, luego del corte que había significado la intervención anterior en 1966. (80)

A continuación Funes agrega, con un grado de detalle que resulta conmovedor, ya que es un índice de hasta qué punto esa «experiencia interrumpida» permanece viva en su recuerdo, como si, en algún punto, continuara siendo ese joven estudiante que asiste con entusiasmo al curso: «Solo se llegaron a dictar los contenidos hasta el punto II.2 y la profesora Lemos había adelantado las clases sobre crítica psicoanalítica, aunque no se había trabajado en las comisiones» (80).

La lectura del programa permite entrever que la concepción que animaba el curso era la de brindar, en las primeras dos unidades, una introducción general y bastante clásica a las distintas corrientes de la teoría literaria (formalismo, estructuralismo, posestructuralismo, crítica psicoanalítica, sociológica, marxista, etc.), para luego pasar a la postulación de una teoría literaria «nacional y popular», «tercermundista». En este sentido, resulta irónico —una amarga ironía histórica que la lectura empática de Funes no destaca-que el programa se haya visto interrumpido en la Unidad II.2 ("Consideración de modelos de análisis literario: Estructuralismo»). Es decir, que no haya llegado a concluir con ese primer tramo de presentación panorámica de teorías literarias, y no haya podido por lo tanto iniciar siquiera el abordaje de un concepto de literatura nacional y popular y de las condiciones específicas para el «estudio literario en el tercer mundo».

Es probable que, de haberse dictado completo, el apoyo teórico fundamental se habría desplazado hacia la crítica marxista y la crítica ideológica, pero lo que se alcanzó a hacer, dejó este otro cuadro: centralidad del estructuralismo checo y centralidad del concepto de modelo. (82)

El comentario de Funes presupone, desde una perspectiva que podríamos caracterizar como típica de la cultura académica (y de la cultura en general) del «Primer mundo», que lo «normal», lo «esperable», lo "preferible» hubiera sido que el curso se hubiera dictado «completo» (y en ese caso, probablemente, el eje del trabajo teórico se hubiera desplazado del estructuralismo a la crítica marxista e ideológica como base para la formulación de una Teoría literaria argentina, tercermundista), pero, justamente, por haber seguido en su formulación este modelo académico «clásico» 
(primero los prolegómenos generales, y solo después, hacia el final del curso, la formulación de una teoría literaria más específica) es que esa teoría nacional y popular no llegó a entrar en escena. Le faltó, podríamos decir, sentido de la oportunidad histórica, una percepción adecuada de la velocidad y la precariedad histórica de los tiempos que les tocaba vivir («La historia nos pasó por encima»), algo que, justamente, su condición de habitantes de un país latinoamericano, tercermundista, acostumbrado a ese tipo de violentos vaivenes y sacudidas e interrupciones, podría haberles dado (les faltó perspectiva tercermundista, podríamos decir con cierta ironía).

Hay un presupuesto común a los trabajos de Funes y Vitagliano, y es la idea de que lo que debería haber sido el «curso normal» de la historia de la teoría literaria en la Argentina se vio afectado - y afectado negativamente - por la dictadura militar. En esta operación de lectura, parten de una certidumbre moral incontestable (el carácter funesto de la dictadura militar que asoló nuestro país entre 1976 y 1983) pero de esa certidumbre derivan otras que me parecen mucho más frágiles. ¿Cómo hubieran sido las cosas de no haber tenido lugar la «interrupción» del orden «normal» de los acontecimientos que implicó supuestamente la Dictadura? Es imposible saberlo, y ese hipotético país contrafáctico sin dudas no sería la Argentina, tal como existió y tal como la conocemos. ¿Qué sentido pueden tener entonces estos ejercicios de rememoración y nostalgia? En muchos casos, lo que se esconde detrás de la operación, apenas velado, es el «rescate» y «relanzamiento» de una determinada agenda que quedó clausurada o relegada. Así, en su ensayo de reconstrucción histórica, Funes señala:

volviendo al curso de Teoría Literaria y las discusiones que generó en las clases y fuera de ellas, creo que es importante tener en cuenta esta diferencia con respecto a las concepciones actuales: el objetivo de máxima era la reflexión y la formulación teórica, de ninguna manera la actividad crítica con los textos literarios. (83)

En este punto crucial, es posible advertir cómo se produce un deslizamiento desde la reseña histórica de un acontecimiento más o menos contingente y anecdótico (la clausura abrupta de la experiencia de este curso en 1974) hacia una evaluación más amplia del devenir histórico ulterior de la disciplina en nuestro país. Que ese es blanco central al que apunta la intervención de Funes queda en evidencia en el final de su texto:

Como bien sabemos, la postura que reivindicaba una especificidad de la teoría no prosperó: fruto de la crisis de los modelos, de las condiciones de un trabajo desde la periferia, lo que se reivindicó como actividad marco ha sido la crítica.' Acepto eso como un desplazamiento histórico, perfectamente explicable, argumentable, entendible. Pero de ningún modo creo que esto implique una superación de la anterior agenda abandonada en esa breve primavera setentista. Los puntos finales del programa citado al comienzo estaban apuntando precisamente a delimitar esa agenda: no la puesta en práctica de una crítica latinoamericana, sino la elaboración misma de una teoría literaria latinoamericana.

El sueño de una «Escuela de Buenos Aires» fue uno de los muchos (y más determinantes para nuestra vida y nuestra historia) que tuvieron una fugaz existencia en aquel lapso primaveral. (83-84)

Funes argumenta que la «anterior agenda abandonada» no fue «superada» por razones estrictamente teóricas, sino que se vio «interrumpida» de manera contingente, por el huracán de la 
historia (primero la Triple A, después el Proceso). Pero, ¿es posible sostener esa distinción entre factores «internos» (necesarios) y «externos» (contingentes)? Lo que subyace es, nuevamente, un preconcepto acerca del modo en que estos procesos de reemplazo de una agenda por otra tienen lugar «normalmente» en las academias del Primer Mundo, según procesos relativamente autónomos y según una lógica «interna» y sin interferencia de factores «externos» (una creencia sobre el modo en que funcionan las cosas «en el Primer Mundo» que se podría relativizar fácilmente, pero no es el punto que nos ocupa). Tampoco parece poder deducirse de la «interrupción abrupta» 0 de la "fugaz existencia», la necesidad de retomar la agenda abandonada en esa breve primavera setentista. Entiendo que en realidad lo que Funes está afirmando es que él desea que esa «agenda abandonada» («la elaboración misma de una teoría literaria latinoamericana») sea retomada. Y ese deseo — como cualquier otro, en principio — es absolutamente válido. Si encuentra aceptación en un grupo lo suficientemente amplio y decidido de personas, podría prosperar. Pero, ¿tiene algún sentido pretender fundamentar, como lo hace Funes en su intervención, ese deseo en acontecimientos históricos? Entiendo que no, y que lo que trata de hacer a través de esa estrategia retórica es darle un matiz de «necesidad» a lo que no es más que contingencia: él desearía que «la postura que reivindicaba una especificidad de la teoría» se hubiera impuesto y hubiera tenido una continuidad en el tiempo, pero no fue eso lo que sucedió. $Y$ afirma que esto sucedió porque la Triple A y la Dictadura alteraron el dictado de los cursos. En otras situaciones históricas ciertas opciones se realizan y otras no por otras razones igualmente contingentes. ¿Es motivo suficiente para postular la necesidad de retomar una vía no continuada? Evidentemente no (de lo contrario, sería "necesario» retomar todas las vías potenciales no efectivizadas en la historia). En realidad, el único motivo por el que un sujeto postula la «necesidad» de retomar una determinada agenda abandonada en el pasado es porque desea hacerlo.

\section{Segunda estación: la primavera alfonsinista y el seminario de Ludmer}

En 2015 se publica la desgrabación del mítico seminario de Josefina Ludmer «Algunos problemas de teoría literaria», editado y prologado por Annick Louis, quien afirma, en la apertura del prólogo, haber tenido la «suerte histórica» de ingresar a la carrera de Letras de la UBA en 1984 lo que le permitió experimentar ese momento marcado por el regreso de la democracia al país y por una intensa renovación institucional en la carrera de Letras. Los mojones de esa renovación (Pezzoni, Panesi, Ludmer, Rosa) son los mismos que señalan Vitagliano y tantos otros. Si en el texto de Funes la figura discursiva fundamental, ya desde el título, es la de la interrupción, en el texto de Louis, como veremos, se establece un juego complejo entre las nociones de continuidad, discontinuidad y acumulación.

Lo primero que señala Louis es lo sorprendente que resulta hoy la intensidad del discurso de Ludmer, su densidad teórica, la calidad de las intervenciones, el nivel de organización del programa y de su equipo de trabajo, teniendo en cuenta que venían de por lo menos una década de interrupción institucional. Pese a que, en ese momento, los protagonistas de la experiencia ponían el acento justamente en la falta de continuidad (Ludmer se lamentaba: «nosotros no tenemos comunidad disciplinaria; nuestra comunidad disciplinaria o es ínfima o está constantemente perturbada por avatares políticos» - 72-), lo que «sorprende y fascina» hoy, por el contrario —anota Louis—, es la «intensidad del discurso». ¿Cómo es posible que, inmediatamente luego de circunstancias tan adversas (es decir, de circunstancias marcadas por una larga etapa de interrupción y 
discontinuidad) un discurso así haya podido emerger? La respuesta de Louis es que lo que hizo posible esto fue que, por debajo de esa interrupción, se había dado un fenómeno de continuidad (oculta, secreta) gracias a la «universidad de las catacumbas» o «universidad paralela». Esa mínima y frágil continuidad, siempre amenazada, es la que habría permitido, sin embargo, durante un «largo período de acumulación», resguardar esos discursos y esos debates teóricos en los grupos privados, que ingresaron a la UBA en el 84 propiciando ese momento fulgurante.

Desde la perspectiva de la historia social, resultó determinante el hecho de que en Buenos Aires, durante la dictadura, algunos intelectuales continuaran sus tareas fuera de la universidad, constituyendo lo que se ha llamado una «universidad de las catacumbas» o "universidad paralela»: grupos de estudio y otros espacios de formación, dirigidos por quienes se vieron obligados a abandonar la universidad pública. La denominación misma de «universidad» para estos grupos indica que quienes los animaron y quienes los frecuentaron consideraban que eran una instancia de formación sistemática, a pesar del marco en que se desarrollaban (generalmente espacios privados). En el momento de la apertura democrática, estos intelectuales estaban listos para reintegrarse a las instituciones y ansiosos por hacerlo; tenían discípulos formados en esos grupos y podían proponer un programa de formación organizado y sólido. (Louis:13-14)

El principal inconveniente que enfrenta una narrativa de estas características, que hace de la idea de continuidad su eje, es que le resulta difícil explicar un fenómeno que, desde su propia lógica, no puede sino presentarse como paradojal. Por un lado, como ya vimos, resulta difícil entender cómo fue posible que, pese $a$ la fuerte interrupción, pese a la fuerte discontinuidad que supuso la dictadura, el momento de refundación de la carrera de Letras de la UBA en los años 1984-1985, y puntualmente el seminario de Ludmer, haya presentado semejante grado de intensidad y de sofisticación teórica. La explicación, como vimos, proviene del reconocimiento, por debajo de esa interrupción, de una continuidad secreta, un proceso subterráneo de trabajo y acumulación que habría podido salir a la luz «recién» con el retorno de la democracia. También Vitagliano señalaba en su trabajo que los textos y autores de la nueva disciplina habían comenzado a circular de manera más o menos subrepticia en la universidad durante los sesenta dentro de cursos que seguían llevando por nombre «Introducción a la Literatura» o «Literatura General», pero que esta práctica, a partir de 1976, «se restringió, en el mejor de los casos, a cursos privados o grupos de estudio» (123124, subrayo yo). Pero si explicar el alto grado de desarrollo alcanzado por la teoría en estas condiciones adversas (y señalo que este carácter «adverso» del trabajo en los grupos de estudio bajo la dictadura es otro axioma incuestionado) resulta difícil, y hay que apelar entonces al carisma o al genio de «héroes modernizadores» como Ludmer, lo que resulta mucho más difícil es explicar por qué luego, en los años transcurridos desde el retorno de la democracia hasta el presente, décadas marcadas por una estabilidad institucional inaudita para los parámetros argentinos, no se produjo sin embargo el esperado florecimiento y consolidación de esa Teoría Literaria Argentina con perspectiva latinoamericana. En este caso no hubo, como en el reseñado por Funes, «primavera interrumpida». Y sin embargo, «desde nuestra perspectiva actual, la de una continuidad establecida precisamente en esa época», una continuidad de treinta años de democracia que significan también treinta años de presencia continua en las instituciones superiores de enseñanza e investigación, Louis confiesa sentir «cierta nostalgia» al leer el seminario. Se reconoce allí una intensidad, agrega, «que resulta de una acumulación de capital — trabajo, lecturas, enseñanza - difícil 
de alcanzar incluso en un sistema [el de la universidad argentina a partir del 84] marcado por la continuidad» (18) y esto, entre otras razones, porque «la topografía académica contemporánea nos sumerge en demandas de producción permanente» (18). Pero entonces, ¿deberíamos concluir que semejante grado de acumulación de capital intelectual es difícil de alcanzar incluso en un sistema marcado por la continuidad o justamente porque nos encontramos dentro de un sistema académico consolidado? ¿No podría deducirse, de los acontecimientos históricos consignados, la interpretación opuesta, esto eso, que los grupos de estudio privados durante la dictadura no constituyeron una "restricción» para el desarrollo de la Teoría sino su ámbito más propicio de florecimiento, quizás porque la dictadura, como estado de excepción permanente y caracterizado por un despliegue inusitado de represión y violencia puede haber alentado, entre los que sostenían el ejercicio de la práctica teórica, la convicción de que defender ese espacio, contra todo y contra todos, era un asunto de la mayor relevancia? ¿No habrá dotado la dictadura al ejercicio de la Teoría de un aura épica de lucha y resistencia ideológico-política que quizás se disolvió paulatinamente en la «normalización» del tiempo histórico posterior? ¿Y entonces no podría afirmarse que acaso fue la interrupción, el cese de las permanentes interrupciones y restricciones institucionales lo que tuvo un efecto «restrictivo» sobre el desarrollo ulterior de una teoría literaria argentina con perspectiva latinoamericana? La aclaración en sí misma es incómoda, pero resulta necesaria: afirmar esto no supone «defender» la dictadura, sino señalar que la presuposición según la cual la lógica de los valores cívicos y las «mejores condiciones de vida» para los ciudadanos es la misma que rige para la producción intelectual y artística es sólo eso, una presuposición sólidamente asentada en valores morales, pero que carece de otro fundamento.

\section{Los índices de institucionalización o cómo el pronóstico del clima siempre puede fallar}

Entiendo que estos trabajos, en su intento de fundamentar objetivamente lo que no son más —ini menos! - que expresiones subjetivas de deseo, también se enfrenta con contradicciones argumentativas con relación al uso que hacen de los «datos». Vuelvo al texto de Louis para ejemplificar este punto. En un primer movimiento de su texto, Louis señala que el Seminario de Ludmer tuvo un carácter absolutamente singular, inaugural, acontecimental (y por lo tanto no mensurable según parámetros normales). En este sentido, cuando introduce algunos datos cuantitativos que, medidos desde los parámetros contemporáneos de eficacia académica, no son muy favorables, inmediatamente aclara que "no es eso lo que importa», ya que claramente "se trataba de otra cosa", algo que escapa a la lógica de las cantidades y responde a la de la intensidad.

Aunque oficialmente solo contara con cincuenta y un inscriptos (de acuerdo a los registros del Departamento de Letras), más de quinientas personas asistieron regularmente (...). Tanto el hecho de que los inscriptos oficiales fueran poco numerosos, como el bajo porcentaje de estudiantes que presentaron el trabajo final para aprobar traducen la función formativa que tuvo este primer seminario de teoría literaria en la UBA y que lo que se venía a buscar era otra cosa. (Louis:15-17)

En este punto, Louis se posiciona al margen de los típicos criterios cuantitativos a los que actualmente se encuentran sometidas las prácticas de enseñanza en marcos institucionales; criterios según los cuales estos indicadores del seminario de Ludmer (pocos inscriptos formales, bajo 
porcentaje de estudiantes que completaron el seminario con su aprobación final) hablarían de algún tipo de falla o ineficacia en su funcionamiento. En este punto Louis argumenta en consonancia con ese otro momento de su texto en el que se lamenta por la «topografía académica contemporánea» que "nos sumerge en demandas de producción permanente» (18) que impiden alcanzar grados de acumulación, intensidad y pasión como los que desplegaron en las clases de Ludmer. Sin embargo, un poco más adelante, al intentar dar pruebas contundentes del «éxito» del proyecto de Ludmer, recurre a un parámetro absolutamente acorde a los de esa misma topografía académica: la presencia de profesores argentinos — formados con Ludmer — en las «comunidades latinoamericanistas» de los grandes centros académicos internacionales:

Percibimos aquí una dimensión del proyecto de Ludmer y otros críticos que se comprometen entonces con la refundación de los estudios literarios: la comunidad argentina aparece en ese momento como marginal, excéntrica, y refundar la carrera significa afirmar una escuela crítica, una escritura y una teoría, y trabajar para convertirla en un centro de producción sobre el hecho literario dotado de un perfil propio. El lugar que la crítica argentina tiene actualmente en las comunidades latinoamericanistas de Estados Unidos y Europa, donde trabajan numerosos colegas argentinos, muestra el éxito del proyecto de estos intelectuales. (23)

Nuevamente, entiendo que hay en este punto un salto difícil de sostener entre un «dato objetivo» (cantidad de «colegas argentinos» que se desempeñan —y se entiende que «exitosamente», quiera decir esto lo que quiera decir - en las «comunidades latinoamericanistas de Estados Unidos y Europa») y lo que no deja de ser la expresión de un deseo (que la carrera de Letras de la UBA se haya convertido, gracias a Ludmer y otros intelectuales, en «un centro de producción sobre el hecho literario dotado de un perfil propio»). El principal problema que encuentro en argumentaciones de este tipo, que hacen coincidir índices de éxito y eficacia institucional (cantidad de académicos argentinos desempeñándose exitosamente en los grandes centros internacionales) con valoraciones más cualitativas y subjetivas sobre el carácter «fundacional» 0 «mítico» o «propiciatorio» del seminario de Ludmer (es decir, que afirman que el Seminario de Ludmer fue una de las causas de ese «éxito» posterior) es que resultan contradictorias con aquellas otras afirmaciones según las cuales, «pese a» las condiciones adversas de la dictadura, la interrupción institucional, etc., luego tuvo lugar un acontecimiento como la primavera del 84-85. Si aceptamos una relación causal entre fenómenos históricos de este tipo, ¿cómo podemos saber cuándo Y sucedió gracias a $\mathrm{X}$ y cuándo lo hizo pese $\mathrm{a} \mathrm{X}$ ? Insisto: entiendo que lo único que nos guía en este tipo de presuposiciones son nuestros valores morales («la Dictadura no puede haber favorecido el florecimiento de la Teoría literaria») pero, en realidad, carecemos de otro fundamento para esta creencia. ${ }^{2}$

\section{Esperando el verano (y a modo de conclusión)}

Creo importante en este punto señalar que no estoy cuestionando las narrativas de Funes y Louis para proponer otra en su lugar. Por el contrario, estoy sugiriendo que no tiene mucho sentido intentar construir una narrativa histórica consistente que permita explicar estos acontecimientos del pasado y deducir de ellos, con cierto fundamento, acciones o lineamientos a seguir. Afirmo que es más sencillo, y más acorde con la incongruencia constitutiva entre los acontecimientos y las narrativas que buscan asignarles un sentido, simplemente desentenderse de esos intentos 
de anclar en una interpretación del pasado los propios deseos contingentes en relación con el presente y el porvenir, y que sería más efectivo simplemente presentarlos como tales. En ese caso, Funes podría decir algo así como «yo querría que se relanzara en el presente un programa de investigación teórica metadiscursiva sobre el objeto literatura con el estructuralismo como base y que apuntara a la fundación de un cuerpo sistemático de pensamiento que recibiera el nombre del "Escuela de Buenos Aires"» y Louis algo así como "yo querría que las discusiones actuales en el campo de la teoría literaria en la universidad argentina tuvieran una densidad conceptual y que produjeran en los interlocutores la sensación de conectarse de manera intensa con los acontecimientos sociopolíticos del presente», y ambos podrían intentar diseminar esos deseos personales entre el mayor número posible de colegas, tratando de convencerlos de que sería hermoso que un proyecto así se llevara a cabo, pero sin remitirlo a una fundamentación en determinada interpretación de algunos momentos míticos del pasado de la teoría literaria en la Argentina.

\section{Notas}

1 Sin dudas uno de los representantes más claros de esta postura ha sido Jorge Panesi: él fue durante muchos años Profesor Titular de la Cátedra de Teoría y Análisis Literario «C» de la UBA y ha producido notables e influyentes intervenciones en el terreno de la crítica literaria, pero no ha acompañado esas intervenciones con una producción de teoría «pura», al menos no según una concepción clásica de la distinción teoría/crítica que siga sosteniendo, en términos de Funes, «la naturaleza específicamente meta-discursiva de la labor teórica» (83). En este contexto se carga de un sentido muy específico y estratégico el título en principio bastante neutro elegido por Panesi para el libro en el que reúne algunos de sus trabajos más destacados: Críticas (2000). En este sentido, la intervención de Funes en las Jornadas de 2009, justamente cuando Panesi había dejado de dirigir el Departamento, puede leerse como una discusión con la concepción no «específicamente meta-discursiva» de la labor teórica que practicó y enseñó Panesi a generaciones de estudiantes.

2 En una línea de análisis muy cercana a la de Annick Louis en este punto - y entiendo que también susceptible de las mismas objeciones- se encuentran los trabajos de Analía Gerbaudo (2011, 2013, 2015) sobre el seminario de Ludmer. No deja de resultar inquietante - al menos desde una perspectiva de lectura como la que este trabajo propone- ese doble movimiento por el que, en los mismos textos en los que se hace un

encomio del lugar fundamental que tuvo un seminario como el de Ludmer, que fue posible luego de un largo período de precariedad institucional, y que se habría constituido como «usina teórica de la universidad argentina posdictadura» (2015) sin que parezca posible decidir si esto sucedió así «pese» o «gracias» a esta precariedad institucional; en esos mismos textos, decíamos, tenga lugar un segundo movimiento según el cual se deduce de determinados "índices de institucionalización» (Gerbaudo 2013:157) la posibilidad de «pronosticar un buen futuro para el área de las Letras en general» (158). Entiendo, por el contrario, que índices de institucionalización altos lo único que pueden pronosticar (de manera circular) es, justamente, un buen futuro para la institucionalización de las Letras como ámbito profesional-laboral, y esto, sin dudas, es un dato positivo desde el punto de vista profesional-laboral (estabilidad, mejores condiciones de trabajo, etc.), pero justamente una experiencia como el seminario de Ludmer, si algo nos indica, es que no sabemos si esas mejores condiciones de institucionalización (estabilidad, continuidad, etc.) permiten pronosticar o no un «buen futuro» para la teoría, la crítica ni para el área de las Letras en general, entendida como un ámbito de producción de acontecimientos imprevistos y renovadores en el pensamiento sobre la literatura, y no de meras repeticiones de lo ya pensado en otro tiempo, en otro lugar. 


\section{Referencias bibliográficas}

Buchbinder, P. (2005). Historia de las universidades argentinas. Buenos Aires: Sudamericana.

Ciordia, M. y otros (2011). Perspectivas actuales de la investigación literaria. Buenos Aires: Editorial de la Facultad de Filosofía y Letras de la Universidad de Buenos Aires.

Eagleton, T. (2003). Después de la teoría. Barcelona: Ramdom House Mondadori.

Ford, A. (2005). 30 años después. 1973: las clases de Introducción a la Literatura y otros textos de la época. Política, Comunicación y Cultura. La Plata: Universidad Nacional de La Plata.

Funes, L. (2009). Teoría Literaria: una primavera interrumpida en los años setenta. Actas de las I Jornadas de Historia de la Crítica en Argentina. Facultad de Filosofía y Letras, Universidad de Buenos Aires, 79-84. www.filo.uba.ar/contenidos/carreras/letras/actas_jornadas

(2011). Medievalismo en el otoño de la Edad Teórica: consideraciones parciales sobre la operación filológica. En M. Ciordia y otros. Perspectivas actuales de la investigación literaria. Buenos Aires: Editorial de la Facultad de Filosofía y Letras de la Universidad de Buenos Aires, 45-78.

Gerbaudo, A. (2011). Al margen de las garantías disciplinares, Josefina Ludmer. Katatay. Revista Crítica Latinoamericana, (9), 83-93.

(2013). Funciones y sentidos de la Teoría literaria. Una conversación entre Josefina Ludmer y Walter Mignolo. Badebec, (5), 155-183.

(2015). Algo más sobre un mítico seminario (usina teórica de la universidad argentina de la posdictadura). $452^{\circ} \mathrm{F},(12), 132-152$.

(2016). Políticas de exhumación: las clases de los críticos en la universidad argentina de la posdictadura 1984-1986. Santa Fe/Buenos Aires: UNL/UNGS.

Lacalle, J. M. y M. Migliore (2015). Aproximaciones a la historia de la Teoría Literaria en la carrera de Letras de la UBA. Parte IV (1976-1985). Luthor, VII(26). www.revistaluthor.com.ar

Louis,A. (2015). Prólogo. En Josefina Ludmer. Clases 1985. Algunos problemas de teoría literaria. Buenos Aires: Paidós, 13-28. Ludmer, J. (2015). Clases 1985. Algunos problemas de teoría literaria. Buenos Aires: Paidós.

Panesi, J. (2000). Críticas. Buenos Aires: Norma.

Peller, D. (2016). Pasiones teóricas. Crítica y literatura en los setenta. Buenos Aires: Santiago Arcos.

(2017). Pasiones y ocasos de la teoría en Argentina. En Dalmaroni, M. y V. Sager, editorxs. La resistencia a la Teoría: Literatura, escritura, lectura. Colección Colectivo Crítico de Libros de la FaHCE, La Plata, Facultad de Humanidades y Ciencias de la Educación, Universidad Nacional de La Plata. En prensa.

Piglia, R. (2016). Las tres vanguardias. Saer, Puig, Walsh. Buenos Aires: Eterna Cadencia.

Riva, G. y J. M. Lacalle (2014a). Aproximaciones a la historia de la Teoría Literaria en la carrera de Letras de la UBA. Parte I (1920-1946). Luthor, IV(19). www.revistaluthor.com.ar (2014b). Aproximaciones a la historia de la Teoría Literaria en la carrera de Letras de la UBA. Parte II (1947-1966). Luthor, IV(20). www.revistaluthor.com.ar (2015). Aproximaciones a la historia de la Teoría Literaria en la carrera de Letras de la UBA. Parte III (1966-1975). Luthor, VI(24). www.revistaluthor.com.ar

Topuzian, M. (2016a). Spoilers de final de temporada. Luthor, (30). www.revistaluthor.com.ar

---. (2016b). Volver al futuro... de la teoría. Exposición leída en Ateneo Permanente. Programa de Estudios Latinoamericanos Contemporáneos y Comparados. 17/08/2016. Mimeo.

Vitagliano, M. (2011). Variaciones sobre un punto: notas de trabajo sobre teoría y crítica literaria. En Martín Ciordia y otros. Perspectivas actuales de la investigación literaria. Buenos Aires: Editorial de la Facultad de Filosofía y Letras de la Universidad de Buenos Aires, 123-154. 